\title{
Sarcopenia May Be a Risk Factor for Osteoporosis in Chinese Patients with Rheumatoid Arthritis
}

\author{
Li Lian',*, Jian-Xiong Wang'*, Yue-Chen $\mathrm{Xu}^{2}$, He-Xiang Zong', Yu-Zhu Teng $\mathbb{D}^{\prime}$, Sheng-Qian Xu' \\ 'Department of Rheumatology \& Immunology, the First Affiliated Hospital of Anhui Medical University, Hefei, 230022, Anhui, People's Republic of \\ China; ${ }^{2}$ Anhui Medical University, Hefei, 230032, Anhui, People's Republic of China \\ *These authors contributed equally to this work \\ Correspondence: Sheng-Qian Xu, Department of Rheumatology \& Immunology, the first Affiliated Hospital of Anhui Medical University, No. 2I 8, Ji-xi \\ Road, Hefei, 230022, Anhui, People's Republic of China, Tel/Fax +86 55I 62922262, Email xushengqianI6@sina.com
}

Purpose: Osteoporosis (OP) has been classically considered a co-morbidity of rheumatoid arthritis (RA). This investigation determined the clinical significance of sarcopenia in patients with RA combined with OP or whether sarcopenia influences RA when combined with OP.

Materials and Methods: Data pertaining to the duration of RA, C-reactive protein (CRP) level, and erythrocyte sedimentation rate (ESR) were collected from 549 RA cases and 158 healthy individuals. Disease activity score at 28 joints (DAS28), the body mass index (BMI), health assessment questionnaire (HAQ), bone mineral density (BMD), and Sharp score were compared between the 2 groups.

Results: The prevalence of OP $\left(33.3 \%\right.$ vs $\left.12.7 \%, \chi^{2}=69.992, \mathrm{P}<0.0001\right)$ and sarcopenia $\left(61.7 \%\right.$ vs $\left.9.0 \%, \chi^{2}=135.336, \mathrm{P}<0.01\right)$ was greater in patients with RA than in healthy controls. RA patients with sarcopenia had a higher incidence of OP at all measured sites than RA patients without sarcopenia (all $\mathrm{P}<0.0001$ ), and the incidence of OP was significantly higher than in patients with mild-tomoderate or severe RA without sarcopenia $(\mathrm{P}<0.0001)$. Differences in age, gender, course of disease, CRP level, ESR, DAS28, BMI, HAQ, BMD, and Sharp score were statistically different between the RA with or without sarcopenia groups $(\mathrm{P}<0.01)$. The incidence of OP and sarcopenia was higher in RA patients treated than not treated with glucocorticoids [GCs] $(36.4 \%$ vs $29.3 \%$, P $<0.05$ and $66.1 \%$ vs $56.0 \%$, respectively; $\mathrm{P}<0.05)$. Logistic regression showed that the risk factors for OP in RA individuals were female $(\mathrm{OR}$, 14.671; 95\% CI, 6.877-31.300; P < 0.0001), age (OR, 1.100; 95\% CI, 1.076-1.124; P < 0.0001), and sarcopenia (OR, 3.561; 95\% CI, 2.214-5.726; P $<0.0001)$.

Conclusion: OP and sarcopenia are common in RA patients. Sarcopenia may be a risk factor for OP occurrence in Chinese patients with RA.

Keywords: rheumatoid arthritis, osteoporosis, sarcopenia, muscle

\section{Introduction}

Osteoporosis (OP) is a common systemic skeletal illness marked by low bone mass and microarchitectural degradation of bone tissue, which leads to bone fragility and fracture susceptibility. ${ }^{1}$ The prevalence of OP in the general population ranges from $9-38 \%$ for women and $1-8 \%$ for males. ${ }^{1}$ The prevalence of OP is estimated to be $>30 \%$ in patients with rheumatoid arthritis (RA), which is a 2 -fold increase over the general population. ${ }^{2,3}$ Furthermore, when compared to people without RA, patients with RA may develop fractures at a higher bone mineral density (BMD). ${ }^{4}$ RA is an inflammatory condition that predisposes patients to develop OP. Moreover, OP in RA patients has a multifactorial pathogenesis in which the use of glucocorticoids (GCs) and systemic inflammation play a major role. ${ }^{5}$ Recent studies have reported an interesting link between RA autoantibodies and the occurrence of OP. ${ }^{1-6}$ Localized bone erosions and general OP are the main features of bone and joint damage in patients with RA. OP is the most common complication in patients with RA, occurring in approximately one-third of patients, ${ }^{7}$ and further resulting in bone fragility and a boosted susceptibility to fractures. ${ }^{8,9}$ 
The mechanism leading to the development of OP in patients with RA has recently been the focus of numerous studies. Increased age, female gender (especially postmenopausal women), low body mass index (BMI), higher disease activity, long-term high-dose steroid therapy, and vitamin D deficiency have been reported to be associated with the development of OP in patients with RA. ${ }^{10-14}$ Hennie et al ${ }^{4}$ concluded that the risk factors for OP in patients with RA include inflammation, use of GCs, immobility, low muscle mass, and sarcopenia.

OP is significantly linked to sarcopenia in the elderly population. ${ }^{15}$ Sarcopenia is characterized by low muscle mass, strength, and function and carries the risk of physical incapacity, low quality of life, and death. ${ }^{16}$ Sarcopenia is classified as primary (or age-related) when no other specific cause is apparent, whereas sarcopenia is classified as secondary when causal elements other than or in addition to aging are present. ${ }^{17}$ Primary sarcopenia occurs most often in the elderly (likely due to age), while secondary sarcopenia most often occurs in youthful individuals with chronic inflammation. ${ }^{16}$ Indeed, patients with RA are at higher risk of developing secondary sarcopenia due to chronic inflammation and joint dysfunction. ${ }^{18}$ Cohort studies have shown that patients with RA have lower muscle mass and the prevalence of sarcopenia ranges from $25.9 \%-43.3 \%{ }^{19-24}$ The wide range in the prevalence of sarcopenia in RA patients reflects differences in the study sample, age, gender, race, and definitions and methods of diagnosing sarcopenia. According to the Japanese database, the prevalence of sarcopenia in RA patients was $28 \% .{ }^{19}$ Tournadre et al ${ }^{25}$ reported that muscle mass is lower in RA patients than the healthy population and the sarcopenia prevalence was higher; however, the relationship between OP and sarcopenia in Chinese patients with RA has rarely been reported. The objective of this study was to determine the relationship between sarcopenia and the incidence of OP in elderly Chinese patients with RA.

\section{Methods}

\section{Participants}

The present retrospective study recruited 549 Chinese patients with RA (age range, 18-86 years) who met the 1987 American College of Rheumatology (ACR) and 2009 European League Against Rheumatism revised RA classification criteria, and 158 gender- and age-matched controls (age range, 24-81 years). They were all inpatients in the Department of Rheumatology of The First Affiliated Hospital of Anhui Medical University between January 2015 and June 2018.

The RA patients included 101 men and 448 women with a disease duration of $10.24 \pm 9.90$ years and an average BMI of $22.4 \pm 3.8 \mathrm{~kg} / \mathrm{m}^{2}$. Healthy individuals from the Department of Rheumatology Physical Examination center served as the control group, and included 30 men and 128 women with a mean BMI of $23.06 \pm 3.04 \mathrm{~kg} / \mathrm{m}^{2}$. Patients with parathyroid gland disease, thyroid disease, severe liver-kidney disorders, endocrinopathies, radiologic irregularities (eg, scoliosis and platyspondyly), and concomitant use of estrogens, androgens, anticonvulsants, or anticoagulants were excluded from this study. Of these patients, 141 had vertebral fractures, but no history of extremity fractures. All RA patients with OP were treated for OP, including calcium with vitamin D.

The study proceeded with the approval of the Ethics Committee at Anhui Medical University, which was in accordance with the ethical standards specified in the 1964 Helsinki Declaration and its later amendments. Informed written consent was achieved from each individual prior to commencing the study.

\section{Clinical and Laboratory Assessment}

The information collected for this research study included patient age and gender, duration of disease, weight, height, GC use, and disease-modifying anti-arthritis drugs (DMARDs). ${ }^{26}$ The disease activity score at 28 joints $($ DAS28) in the RA group was calculated as follows: DAS28 $(\mathrm{ESR})=(0.56 * \operatorname{sqr}[\mathrm{TJC}])+(0.28 * \operatorname{sqr}[\mathrm{SJC}])+(0.7$ $* \ln [\mathrm{ESR}]+[0.014 * \mathrm{GH}])$, where $\mathrm{TJC}=$ tender joint count and $\mathrm{SJC}=$ swollen joint count. ${ }^{27,28}$ The following data were also collected for RA patients: ESR (mm/h); CRP $(\mathrm{mg} / \mathrm{L})$; health assessment questionnaire (HAQ) score; Sharp score; rheumatoid factor (RF in $\mathrm{IU} / \mathrm{mL}$ ); and anti-citrullinated peptide antibodies (ACPA in IU/mL). ESR was measured using the employing modified Westergren method, ACPA, CRP, and RF were measured using the immunoturbidimetric method. Based on the DAS28 score, RA was divided into two groups: mild-to-moderate disease activity (DAS28<5.1); and severe disease activity (DAS28 $>5.1$ ). Dressing, arising, eating, walking, hygiene, 
reach, grasp, and activities are the eight sections of the HAQ. Each segment has two or three questions. Within each section, the scoring ranges from 0 (no difficulty) to 3 (unable to do). The score assigned to each part is the lowest score within the section, ie, if 1 question is rated 1 and another question is rated 2, the section score is 2 . The pain visual analog scale (VAS) was completed by RA patients. The pain VAS is a single-item scale that typically ranges from "no pain" (score of 0) to "worst imagined pain" (score of 10). The RA patients completed the pain VAS independently. The respondent was asked to draw a line perpendicular to the VAS line at the point where the pain intensity was the greatest. A higher pain intensity score implies more discomfort, as follows: no pain $(0-0.4 \mathrm{~cm})$; mild pain $(0.5-4.4 \mathrm{~cm})$; moderate pain $(4.5-7.4 \mathrm{~cm})$; and severe pain $(7.5-10 \mathrm{~cm}){ }^{29,30}$

\section{Measurements of BMDs}

Dual-energy X-ray absorptiometry (DEXA) was performed to determine the bone mineral density (BMD) in the lumbar spine 1-4 (L1-4), femoral neck (neck), and total hip (hip) using a Lunar Prodigy DF +310504 (GE Healthcare, USA). BMD T scores were calculated from the number of standard deviations. Assuming that the SD of BMD was $0.12 \mathrm{~g} / \mathrm{cm}^{2}$, it can be inferred that the peak BMD was 0.90 and $0.95 \mathrm{~g} / \mathrm{cm}^{2}$ in women and men, respectively. All of the densitometry procedures were completed according to the manufacturer's instructions for spinal BMD measurements. Patients with a T-score $\leq-2.5$ and premenopausal women or men $<50$ years of age with a z-score $\leq 2$ were diagnosed with OP according to the WHO classification. ${ }^{31}$

\section{Diagnosis of Sarcopenia}

Skeletal muscle mass was determined by bioelectrical impedance analysis [BIA] (Inbody 720 human component analyzer; South Korea) in 549 RA patients and 156 controls (2 patients were not tested due to immobility). A strong correlation between BIA resistance and SM measurements in the arms and legs has been previously reported. ${ }^{32,33}$ Sarcopenia was diagnosed based on the Asia Working Group for Sarcopenia (AWGS) criteria. ${ }^{34}$ The skeletal muscle index (SMI) was computed as follows: skeletal muscle mass $/$ height ${ }^{2}\left(\mathrm{~g} / \mathrm{m}^{2}\right)$. Patients with RA were divided into two groups (RA with and without sarcopenia).

\section{Statistical Analysis}

Continuous data obtained from the study are presented as the mean $\pm \mathrm{SD}$, and categorical variables are presented as the median (P25-P75). Normally-distributed continuous data were assessed using Student's $t$-test and the Mann-Whitney $U$-test. Chi-square analysis was used to evaluate categorical variables. Risk factors for OP were identified by multivariable logistic regression. Statistical analyses were performed using SPSS (version 23.0). The test of significance was a 2-tailed test at an $\alpha=0.05$.

\section{Results}

There were no statistical differences in age, BMI, and gender between the RA and control groups $(P>0.05)$.

\section{Comparison of the Prevalence of OP and Sarcopenia Between the RA and Control Groups}

The BMD at all the measured sites in RA patients decreased significantly compared with the controls $(\mathrm{P}<0.0001)$. The OP rate in patients with RA was 33.3\% (183/549), which was significantly higher than the OP rate in the controls (20/158 $\left.[12.7 \%] ; \chi^{2}=69.992, \mathrm{P}<0.0001\right)$. The incidence of lumbar (L1-4) and total hip OP in RA patients was $18.2 \%$ and $16.0 \%$, respectively, which was greater than the corresponding incidence in the control group $(\mathrm{P}<0.0001)$. The SMI was significantly lower in RA patients than in controls $(\mathrm{P}=0.0001)$. The incidence of sarcopenia in patients with RA was $61.7 \%$ (339/549), which was higher than controls (14/156 [9.0\%]; $\chi^{2}=135.336, \mathrm{P}<0.0001$; Table 1 and Figure 1).

Disease activity according to the DAS28 score was defined as follows: $<3.2$, low disease activity; $3.2-5.1$, moderate disease activity; and > 5.1, severe activity. Among 549 patients with RA, 260 had low-to-moderate disease activity and 289 had severe disease activity. Seventy-five patients with low disease activity had OP (28.8\% [75/260]). In the severe 
Table I Characteristics Between RA and Controls

\begin{tabular}{|l|l|l|l|}
\hline Indicators & RA $(\mathbf{n}=549)$ & Controls $(\mathbf{n}=\mathbf{I 5 8})$ & $\mathbf{P}=$ \\
\hline Age $($ year) & $55.6 \pm 13.3$ & $53.9 \pm 12.5$ & 0.137 \\
Female, $\mathrm{n}(\%)$ & $448(81.6 \%)$ & $128(81.0 \%)$ & 0.882 \\
BMI $\left(\mathrm{kg} / \mathrm{m}^{2}\right)$ & $22.4 \pm 3.8$ & $23.06 \pm 3.04$ & 0.099 \\
BMD-Neck $\left(\mathrm{g} / \mathrm{cm}^{2}\right)$ & $0.79 \pm 0.16$ & $0.92 \pm 0.15$ & 0.0001 \\
BMD-Hip $\left(\mathrm{g} / \mathrm{cm}^{2}\right)$ & $0.82 \pm 0.16$ & $0.97 \pm 0.15$ & 0.0001 \\
BMD-LI $\left(\mathrm{g} / \mathrm{cm}^{2}\right)$ & $0.88 \pm 0.16$ & $1.03 \pm 0.19$ & 0.0001 \\
BMD-L2 $\left(\mathrm{g} / \mathrm{cm}^{2}\right)$ & $0.94 \pm 0.18$ & $1.07 \pm 0.19$ & 0.0001 \\
BMD-L3 $\left(\mathrm{g} / \mathrm{cm}^{2}\right)$ & $1.02 \pm 0.19$ & $1.14 \pm 0.19$ & 0.0001 \\
BMD-L4 $\left(\mathrm{g} / \mathrm{cm}^{2}\right)$ & $1.03 \pm 0.19$ & $1.13 \pm 0.18$ & 0.0001 \\
BMD-LI-4 $\left(\mathrm{g} / \mathrm{cm}^{2}\right)$ & $1.00 \pm 0.18$ & $1.11 \pm 0.18$ & 0.0001 \\
SMI $\left(\mathrm{kg} / \mathrm{m}^{2}\right)$ & $5.64 \pm 1.09$ & $8.67 \pm 1.43$ & 0.0001 \\
\hline
\end{tabular}

Abbreviations: BMI, body mass index; BMD, bone mineral density; SMI, skeletal muscle index.

disease activity group, 108 patients had OP (37.4\% [108/289]). The incidence of OP in the high disease activity group was higher than the low disease activity group $\left(\chi^{2}=4.475, \mathrm{P}=0.037\right.$; Figure $\left.1 \mathrm{~B}\right)$.

\section{Disease Characteristics Between RA Patients with and without Sarcopenia}

Age, disease duration, ESR, CRP level, DAS28 score, and HAQ and Sharp scores were significantly higher in RA patients with sarcopenia compared to RA patients without sarcopenia $(\mathrm{P}<0.01)$. The BMD of all measurement sites was significantly lower in RA patients with sarcopenia than RA patients without sarcopenia $(\mathrm{P}<0.01$; Table 2). The percentage of patients with mild disease activity (7.1\% [15/210]), moderate disease activity (47.1\% [99/210]), and severe disease activity (45.7\% [96/210]) were different in the RA without sarcopenia group than the RA with sarcopenia group $\left(5.3 \%, 37.8 \%\right.$, and $56.9 \%$, respectively; $\left.\chi^{2}=6.587, \mathrm{P}<0.05\right)$. The proportion of RA patients with sarcopenia who were treated with GCs was $61.7 \%$, which was higher than RA patients without sarcopenia $\left(51.0 \% ; \chi^{2}=5.790, \mathrm{P}<0.05\right)$. In the group with sarcopenia, $41.6 \%$ of RA patients were treated with DMARDs compared to $44.3 \%$ of RA patients without sarcopenia $(\mathrm{P}>0.05)$. The mean BMI was decreased in patients with sarcopenia $(\mathrm{P}<0.01)$. There were no significant differences in joint tenderness counts, joint swelling counts, VAS scores, and RF and ACPA titers between RA patients with and without sarcopenia. The disease characteristics of the two groups are shown in Table 2.

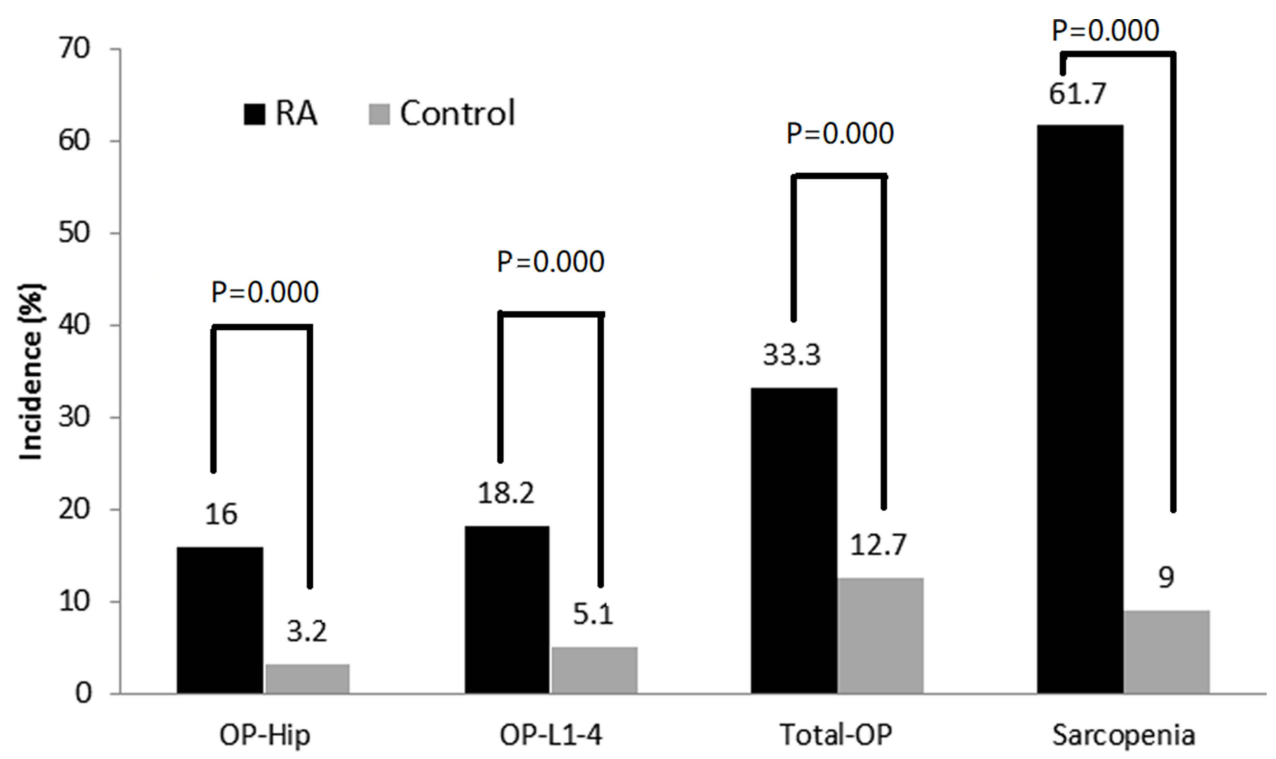

Figure I Comparison of incidence about osteoporosis and sarcopenia between rheumatoid arthritis and control. 
Table 2 Disease Characteristics Between RA Individuals with and without Sarcopenia

\begin{tabular}{|c|c|c|c|c|}
\hline Indicators & RA without Sarcopenia $(n=2 \mid 0)$ & RA with Sarcopenia $(n=339)$ & $\mathbf{Z} / \mathbf{t} / \chi^{2}$ & $\mathbf{P}=$ \\
\hline Age (years) & $54.04 \pm 11.29$ & $58.67 \pm 12.90$ & 5.697 & 0.0001 \\
\hline Female (\%) & $159(75.7 \%)$ & $310 / 339(91.5 \%)$ & 20.313 & 0.0001 \\
\hline BMI $\left(\mathrm{kg} / \mathrm{m}^{2}\right)$ & $24.19 \pm 3.42$ & $20.90 \pm 3.30$ & 4.067 & 0.0001 \\
\hline Disease Duration (years) & $7(2-17.25)$ & II (4-20) & 2.683 & 0.005 \\
\hline VAS $(\mathrm{cm})$ & $5.09 \pm 1.94$ & $5.31 \pm 2.00$ & 1.247 & 0.213 \\
\hline SJC & $6.0(3.0-11.7)$ & $7.0(4.0=12.0)$ & 0.888 & 0.374 \\
\hline TJC & $9.5(5.25-16.0)$ & $12.0(6.5-20.5)$ & 1.879 & 0.060 \\
\hline Morning stiffness (min) & $0(0-30)$ & $0(0-10)$ & 2.380 & 0.812 \\
\hline $\operatorname{ESR}(\mathrm{mm} / \mathrm{h})$ & $54.50(30.0-77.75)$ & $66(46.50-88.50)$ & 3.252 & 0.001 \\
\hline CRP $(\mathrm{mg} / \mathrm{l})$ & $21.48(8.42-54.83)$ & $32.29(14.22-62.89)$ & 2.399 & 0.016 \\
\hline DAS28 & $5.02 \pm 1.21$ & $5.29 \pm 1.20$ & 2.753 & 0.006 \\
\hline HAQ & $1.04 \pm 0.74$ & $1.44 \pm 2.02$ & 2.785 & 0.006 \\
\hline $\mathrm{RF}(\mathrm{U} / \mathrm{mL})$ & $99.10(24.10-271.88)$ & $98.0(26.70-254.5)$ & 1.801 & 0.072 \\
\hline ACPA $(R U / m L)$ & $284.5(72-820.5)$ & $352.0(99.5-964.5)$ & 1.930 & 0.054 \\
\hline Sharp'score & $38(10-77.25)$ & $74(19.00-156.5)$ & 4.873 & 0.0001 \\
\hline BMD-Neck $\left(\mathrm{g} / \mathrm{cm}^{2}\right)$ & $0.84 \pm 0.14$ & $0.75 \pm 1.16$ & 6.797 & 0.0001 \\
\hline BMD-Hip $\left(\mathrm{g} / \mathrm{cm}^{2}\right)$ & $0.88 \pm 0.14$ & $0.77 \pm 0.15$ & 8.964 & 0.0001 \\
\hline BMD-LI $\left(\mathrm{g} / \mathrm{cm}^{2}\right)$ & $0.94 \pm 0.17$ & $0.85 \pm 0.15$ & 6.164 & 0.0001 \\
\hline BMD-L2 $\left(\mathrm{g} / \mathrm{cm}^{2}\right)$ & $1.00 \pm 0.16$ & $0.89 \pm 0.18$ & 7.853 & 0.0001 \\
\hline BMD-L3 $\left(\mathrm{g} / \mathrm{cm}^{2}\right)$ & $1.09 \pm 0.17$ & $0.96 \pm 0.19$ & 8.004 & 0.0001 \\
\hline BMD-L4 $\left(\mathrm{g} / \mathrm{cm}^{2}\right)$ & $1.11 \pm 0.17$ & $0.98 \pm 0.18$ & 7.73 & 0.0001 \\
\hline BMD-LI-4 (g/cm $\left.{ }^{2}\right)$ & $1.07 \pm 0.16$ & $0.95 \pm 0.18$ & 7.657 & 0.0001 \\
\hline GCs user (\%) & 107 (51.0\%) & 209 (61.7\%) & 5.790 & 0.017 \\
\hline Does of GCs/D & $3.86 \pm 4.53$ & $5.48 \pm 5.38$ & 3.071 & 0.002 \\
\hline DMARDs user (\%) & $93(44.3 \%)$ & $|4|(4 \mid .6 \%)$ & 0.338 & 0.594 \\
\hline
\end{tabular}

Abbreviations: BMI, body mass index; VAS, visual analog scale; SJC, swollen joint count; TJC, tender joint count; ESR, erythrocyte sedimentation rate; CRP, C-reactive protein; DAS28, disease activity score at 28 joints; HAQ, health assessment questionnaire; RF, rheumatoid factor; ACPA, anti-citrullinated peptide antibodies; BMD, bone mineral density; GCs, glucocorticoids; DMARDs, disease-modifying anti-arthritis drugs.

\section{Comparison of the Prevalence of OP Between RA Patients with and without Sarcopenia and Between Groups with Different Disease Activities}

In the group of RA patients with sarcopenia ( $\mathrm{n}=339)$, the hip, L1-4, and total OP rates were $22.4 \%$ (76/339), 24.8\% (84/ $339)$, and 43.4\% (147/339), respectively, which were significantly higher than RA patients without sarcopenia $(5.7 \%$ [12/ 210], $\chi^{2}=61.057, \mathrm{P}<0.0001 ; 7.6 \%[16 / 210], \chi^{2}=54.245, \mathrm{P}<0.0001 ;$ and $17.1 \%[36 / 210], \chi^{2}=49.593, \mathrm{P}<0.0001$; Figure 2A). The RA group was further divided into two groups based on the DAS28 scores: mild-to-moderate disease activity group (DAS28 $\leq 5.1, \mathrm{n}=260$ ); and severe disease activity group (DAS28 $\geq 5.1, \mathrm{n}=289$ ). The incidence of OP was higher in the severe disease activity group than the mild-to-moderate disease activity group (37.4\% [108/289] vs $28.8 \%$ [75/260], $\chi^{2}=4.475, \mathrm{P}=0.034$; Figure 2B).

\section{Stratified Analysis of OP Incidence Between RA Groups with Different Disease Activities as a Function of Sarcopenia Status}

Among RA patients without sarcopenia $(\mathrm{n}=210)$, the incidence of OP was higher in those with severe disease activity $\left(22.9 \%\right.$ [22/96]) than mild-to-moderate disease activity $\left(12.3 \%\right.$ [14/114]; $\left.\chi^{2}=4.150, \mathrm{P}=0.042\right)$. Among RA patients with sarcopenia $(\mathrm{n}=339)$, there was no major difference in the OP rate between those with mild-to-moderate $(41.8 \%$ [61/146]) and severe disease activity (44.6\% [86/193]; $\left.\chi^{2}=0.261, \mathrm{P}=0.609\right)$. The OP rate was higher in RA patients with sarcopenia than RA patients without sarcopenia in those with mild-to-moderate and severe disease activity $\chi^{2}=27.142, \mathrm{P}<0.0001$; and $\chi^{2}=12.831, \mathrm{P}<0.0001$, respectively; Figure 3). 


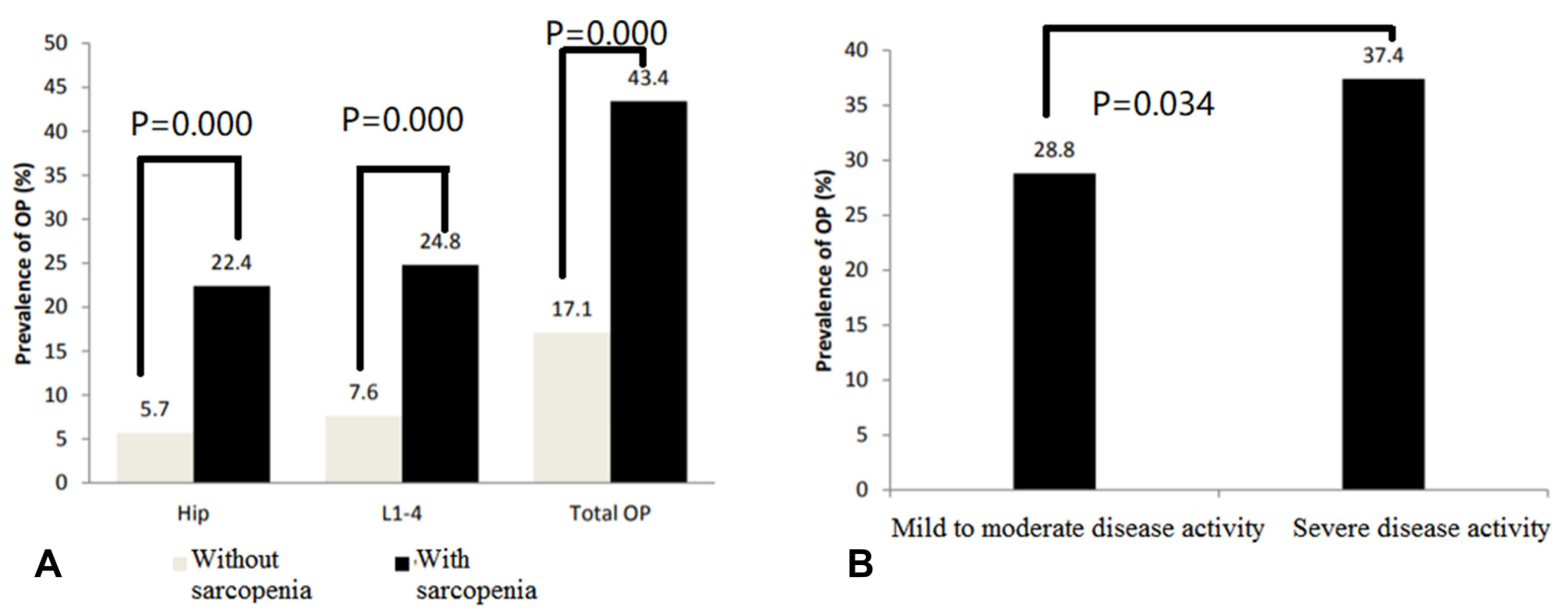

Figure 2 (A) Comparison of incidence of osteoporosis between rheumatoid arthritis patients with and without sarcopenia; (B) Comparison of incidence of osteoporosis between patients with mild to moderate disease activity and with severe disease activity.

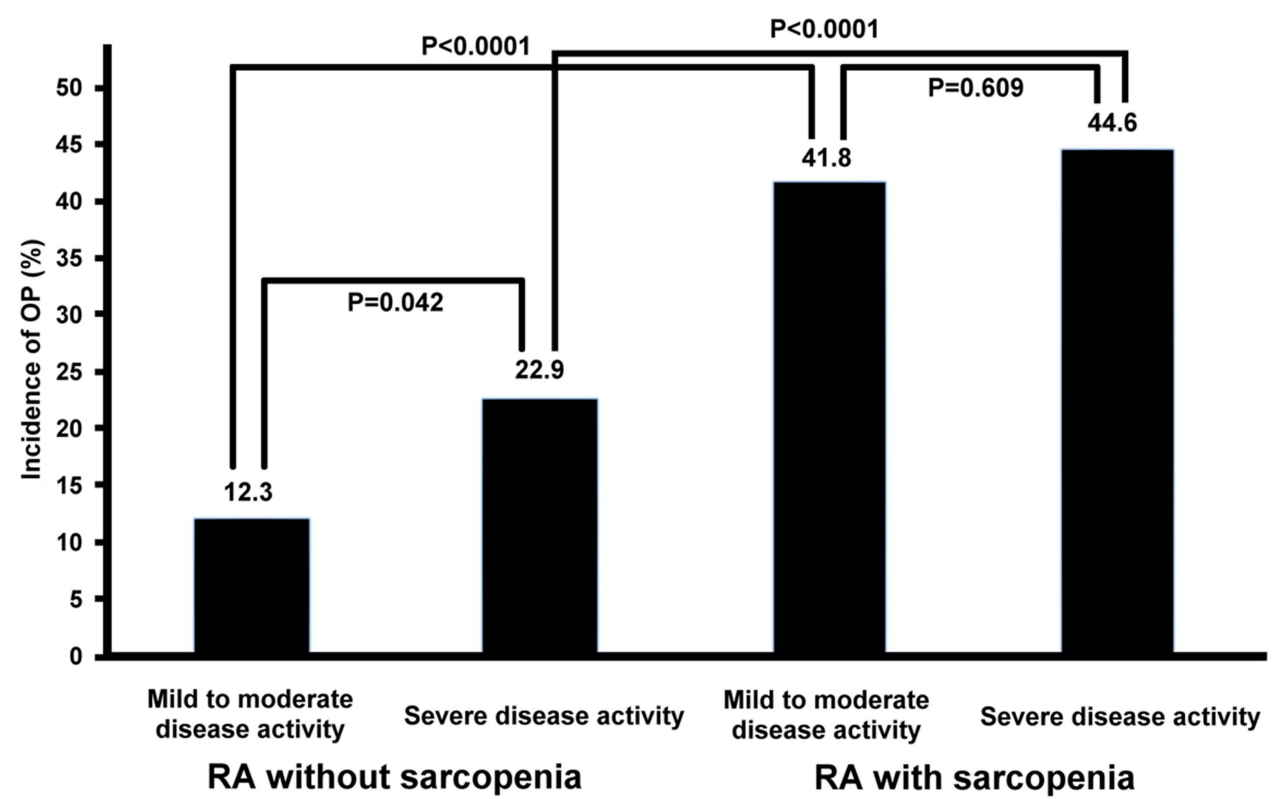

Figure 3 Stratified analysis of osteoporosis incidence between rheumatoid arthritis groups with different disease activity by sarcopenia status.

\section{Prevalence of OP and Sarcopenia in RA Patients Who Did and Did Not Use GCs}

A total of 316 patients were treated with GCs in the current study. The incidence of OP was $36.4 \%(115 / 316)$ in RA patients who used GCs, which was higher than RA patients who were not using GCs $\left(29.3 \%[68 / 232] ; \chi^{2}=8.031\right.$, $\mathrm{P}<0.05)$. The prevalence of sarcopenia was higher in the RA group who used than did not use GCs $(209 / 316$ [66.1\%] vs 130/232 [55.8\%]; $\chi^{2}=5.790, \mathrm{P}<0.05 ;$ Figure 4).

\section{Logistic Regression of OP Risk Factors in Patients with RA}

Multivariable logistic regression was used to determine the prospective risk factors for OP in patients with RA. Age, gender, disease duration, use of GCs, DAS28 score, and sarcopenia were defined as independent variables, and RA with OP was defined as the dependent variable. These analyses showed that the risk factors for OP in RA patients were as 


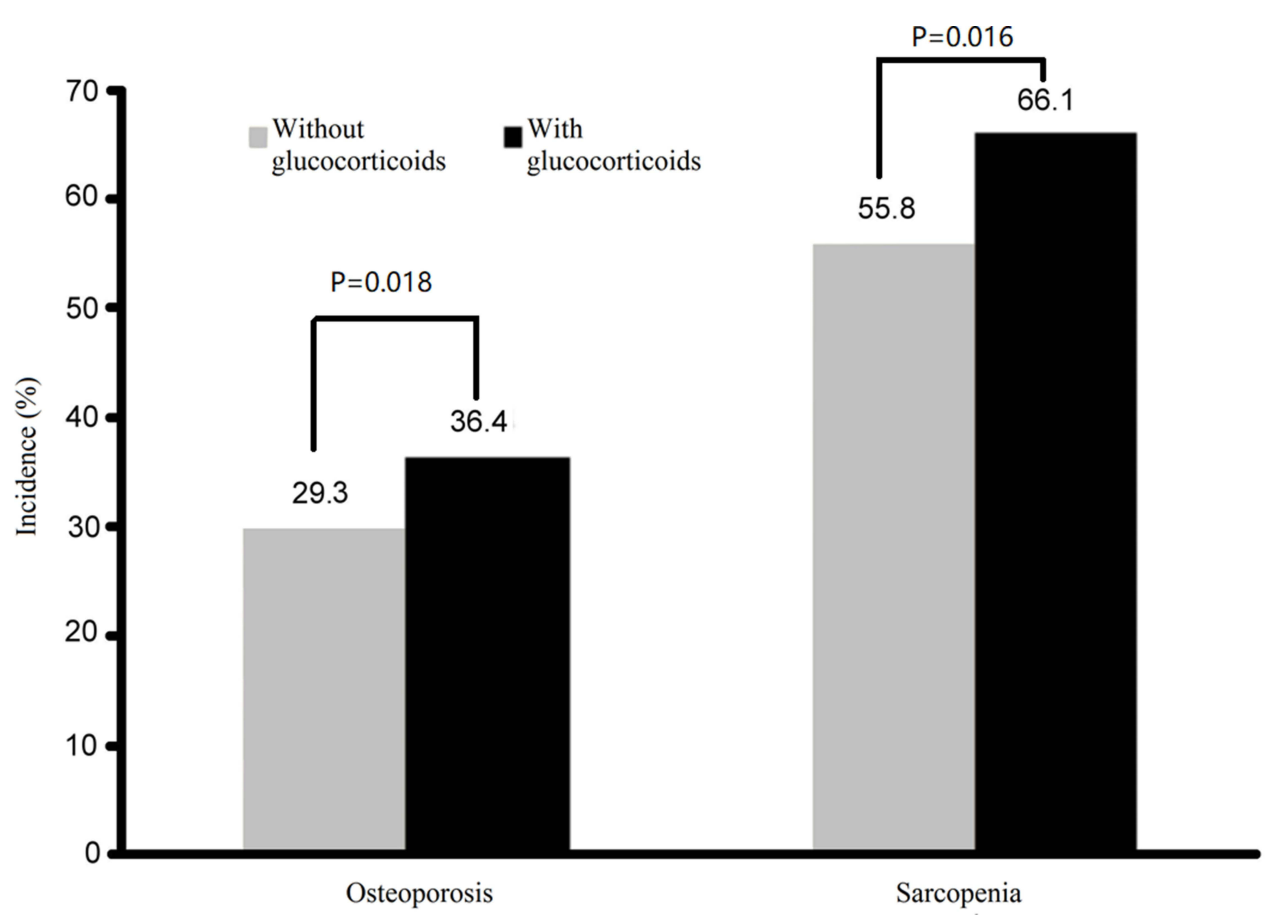

Figure 4 Incidences of osteoporosis and sarcopenia in rheumatoid arthritis patients with and without glucocorticoids.

follows: female (OR, 14.671; 95\% CI, 6.877-31.300; $\mathrm{P}<0.0001)$; age (OR, 1.100; 95\% CI, 1.076-1.124; $\mathrm{P}<0.0001)$; and sarcopenia (OR, 3.561; 95\% CI, 2.214-5.726; P<0.0001).

\section{Discussion}

In the current study we showed that the prevalence of OP was $33.3 \%$ in RA patients and $12.7 \%$ in controls. In addition, we showed that RA patients with severe disease activity had higher OP and GC use rates. OP can develop in RA patients through different mechanisms, including the direct effects of RA, the use of GCs, and estrogen deficiency during the menopause. The prevalence of OP was 3.5 times higher in RA patients with sarcopenia than in RA patients without sarcopenia (OR, 3.561; $\mathrm{P}<0.0001)$. Of these patients, 141 had vertebral fractures, but no history of extremity fractures. All RA patients with OP were treated for OP, including calcium with vitamin D.

Previous studies reported that approximately one-third (32.3\%) of RA patients have bone loss, and the rate of spinal or hip OP was considerably higher in men with RA than in controls. ${ }^{35,36} \mathrm{OP}$ in RA patients might be explained by several factors, including lower muscle mass and sarcopenia. ${ }^{4,9-14}$ Recently, Barone et al ${ }^{17}$ reported that the prevalence of sarcopenia in Caucasians with RA was 21\%, which was less compared to Asian patients with RA (37.1\%) using the same methodological approach. ${ }^{20}$ Of note, patients enrolled in the Barone et al study ${ }^{17}$ were younger compared to the Torii $\operatorname{study}^{20}$ (median was 5 years old, [age range, 48.0-62.2 years] vs [age range, 54.2-72.0 years]).

The sarcopenia rate in RA patients was $61.7 \%$, which is a higher rate than previously reported. ${ }^{27,28}$ We used the cutoff values for AWGS as in the two reports. ${ }^{27,28}$ The median age was 55.6 years (age range, 16-86 years), which is similar to Michael. Furthermore, $47.3 \%$ of the 76 RA patients were in disease remission, as assessed by DAS28, in the Michael study. In the current study, however, the majority of enrolled RA patients were in active status (DAS28 $\geq 3.2$ [ $n=516$ $\{94.0 \%\}]$ ). Thus, different disease activity status and ethnicity may partially explain the differences in prevalence.

Factors associated with sarcopenia have been described previously in patients with RA. Specifically, Giles et al ${ }^{21}$ and Dogan et $\mathrm{al}^{22}$ reported that the percentage of sarcopenia patients with elevated CRP levels $(>0.8 \mathrm{mg} / \mathrm{dL})$ was notably greater than patients without sarcopenia. Higher RF titers, higher HAQ scores, and joint deformity scores are all associated with sarcopenia. ${ }^{21}$ Additionally, lower BMI and cardiovascular risk are also potential risk factors for sarcopenia. ${ }^{19,23}$ Torii et $\mathrm{al}^{20}$ reported that age (61 years; IOR, 49.3-69.0, $\mathrm{P}<0.001$ ), longer disease duration (19 years; 
IOR, 11.0-29.8, $\mathrm{P}<0.001$ ), disease activity of RA assessed by DAS28 (3.2; IOR, 2.5-3.9, $\mathrm{P}<0.001$ ), and GC use (42.4\% vs $25.0 \%, \mathrm{P}<0.001$ ) were all associated with sarcopenia. ${ }^{20}$ An average GC dosage $\geq 3.25 \mathrm{mg}$ /day was a significant factor for developing sarcopenia. ${ }^{37}$ The proportion of GC users and the average daily GC dose were higher in the sarcopenia group than the non-sarcopenia group.

Our results showed that RA patients with sarcopenia have higher disease activity than RA patients without sarcopenia. Also, our data revealed that the proportion of women, age, disease duration, HAQ score, ESR, CRP level, and Sharp score were substantially higher in RA patients with sarcopenia than RA patients without sarcopenia; however, sarcopenia was accompanied by a lower BMI, which is consistent with the reports by Ngeuleu ${ }^{23}$ and Tada. ${ }^{19}$ A recent study suggested that bDMARDs could improve muscle mass in RA individuals. ${ }^{24}$ Torii et al $^{20}$ also reported that a lower percentage of patients in the sarcopenia group were treated with biologic (b)DMARDs compared to non-sarcopenia patients $(32.6 \%$ vs $48.8 \%, \mathrm{P}=0.002)$. In the current study the percentage of patients treated with bDMARDs was also lower in the sarcopenia group than the non-sarcopenia group, while there were no major differences between the two groups.

Sarcopenia and OP share a number of common pathways, including increased inflammatory cytokine activity, sensitive reduction in anabolic hormone secretion, anabolic or myokines and osteokines released from skeletal muscle or bone cells, and eventually a decline in physical activity. Sarcopenia reduces activities of daily living, leading to OP, falls, fractures, and deaths. ${ }^{37-40}$ Sarcopenia is an independent risk factor for hospitalization, OP, cardiovascular diseases, and mortality. ${ }^{11}$ Yoon ${ }^{41}$ showed that sarcopenia is significantly more common in hip, vertebral, and distal radius fractures $\left(41.5 \%, 35 \%\right.$, and $29.6 \%$, respectively) with a prevalence of $35.8 \%(39 / 108)$. Lee et al ${ }^{42}$ also reported that sarcopenia is an independent risk factor for decreased BMD in patients with chronic obstructive pulmonary disease (OR, 6.952; 95\% CI, 3.418-14.139; $\mathrm{P}<0.001)$. In a cross-sectional study, Di Monaco et al ${ }^{43}$ analyzed 313 elderly women with recent hip fractures and analyzed the relationship between sarcopenia and OP. Of the 313 women, 58\% were diagnosed with sarcopenia and $74 \%$ with OP. A binary logistic regression analysis revealed a high prevalence of both complications and a considerable connection between sarcopenia and $\mathrm{OP}(\mathrm{P}=0.026)$.

In addition, women with sarcopenia had a 1.8 -fold increased probability of OP (95\% CI, 1.07-3.02) based on multivariable analysis. Older age and lower BMD were also shown to be associated with hip fractures in Japanese patients $(\mathrm{OR}=1.103,0.082$, and 1.476; $\mathrm{P}<0.001) .{ }^{44}$ In the current study, the BMDs at all measured sites were lower in the sarcopenia group than the non-sarcopenia group. Moreover, the incidence of OP in the lumbar spine and hips was elevated in the sarcopenia group compared to the non-sarcopenia group.

A stratified analysis showed that the prevalence of OP was higher in RA patients with severe disease activity than RA patients with mild-to-moderate disease activity, independent of the existence of sarcopenia; however, the difference was significant only in the RA group without sarcopenia. We conclude from these results that sarcopenia plays a role in patients with RA with OP rather than disease activity. Moreover, results of logistic regression analysis confirmed our conclusion that sarcopenia is a risk factor for OP in patients with RA.

Due to reduced physical activity, ${ }^{45}$ increased concentrations of TNF- $\alpha$ and IL-1 $\beta$, high CRP levels, increased energy expenditure at rest, and inactivity secondary to stiffness and pain, RA individuals are at increased risk for sarcopenia. ${ }^{46}$ Exercise remains the measure of choice for the management of sarcopenia. The role of nutrition in the prevention and treatment of sarcopenia is unclear, but ensuring adequate protein intake and supplementation with deficient nutrients and vitamins is recommended. ${ }^{47}$

The limitations of this study were as follows. First, we used the AWGS criteria to assess sarcopenia in patients with RA, while the AWGS criteria have not been verified for the assessment of secondary sarcopenia, such as RA. Because the main purpose of the EWGOP or AWGS diagnostic criteria is to assess primary sarcopenia, which is an age-related decline in muscular mass, strength, and physical function, it has not been established how aging and RA make a difference with respect to sarcopenia. Second, DMARDs include traditional synthetic DMARDs (csDMARDs), bDMARDs, and targeted synthetic DMARDs (tsDMARDs). In this study, RA patients were treated with bDMARDs; a subgroup analysis of DMARDs (csDMARDs, bDMARDs, and tsDMARDs) should be performed in the future to determine the effect of different DMARDs therapies on the incidence of sarcopenia. Third, there may have been a selection bias in this study because the subjects with RA were active, which may have contributed to a higher 
prevalence of sarcopenia. Also, because the hands and knee joints of patients with RA were swollen and painful, and the joint functions were poor, it was difficult to check the grip strength and gait speed, which may add some limitations of the data. Determining the mechanisms underlying the sarcopenia-OP association in RA individuals and developing effective preventive therapies will require further research. As well, further longitudinal studies are needed to understand the role of RA patient's ageing in the development of sarcopenia.

In summary, sarcopenia is a risk factor for OP in Chinese patients with RA. Sarcopenia is closely related to OP, and the combined effect of both may exacerbate the adverse outcomes in RA individuals. Preventing sarcopenia is as important as preventing OP in patients with RA.

\section{Data Sharing Statement}

Upon reasonable request, all unidentified data related with this study can be obtained from the corresponding author.

\section{Ethics Approval}

The study was proceeded with the approval of the Ethics Committee of Anhui Medical University.

\section{Consent to Participate}

Written informed consent was obtained from all individuals who participated in the study.

\section{Funding}

There is no funding to report.

\section{Disclosure}

The authors declared of no competing interests.

\section{References}

1. Wade SW, Strader C, Fitxpatrick LA, Anthony MS, O’Malley CD. Estimating prevalence of osteoporosis: examples from industrialized countries. Arch Osteoporos. 2014;9:182. doi:10.1007/s11657-014-0182-3

2. Haugeberg G, Uhlig T, Falch JA, Halse JI, Kvien TK. Bone mineral density and frequency of osteoporosis in female patients with rheumatoid arthritis: results from 394 patients in the Oslo County Rheumatoid. Arthritis Register Arthritis Rheum. 2000;43:522-530. doi:10.1002/15290131(200003)43:3<522::AID-ANR7 7 3.0.CO;2-Y

3. Hauser B, Riches PL, Wilson JF, Horne AE, Ralston SH. Prevalence and clinical prediction of osteoporosis in a contemporary cohort of patients with rheumatoid arthritis. Rheumatology. 2014;53:1759-1766. doi:10.1093/rheumatology/keu162

4. Raterman HG, Lems WF. Pharmacological management of osteoporosis in rheumatoid arthritis patients: a review of the literature and practical guide. Drugs Aging. 2019;36:1061-1072. doi:10.1007/s40266-019-00714-4

5. Llorente I, García-Castañeda N, Valero C, González-álvaro I, Castañeda S. Osteoporosis in rheumatoid arthritis: dangerous liaisons. Front Med (Lausanne). 2020;7:601618. doi:10.3389/fmed.2020.601618

6. Adami G, Saag KG. Osteoporosis pathophysiology, epidemiology, and screening in rheumatoid arthritis. Curr Rheumatol Repn. 2019;21:34. doi:10.1007/s11926-019-0836-7

7. Tong JJ, Xu SQ, Zong HX, Pan MJ, Teng YZ, Xu JH. Prevalence and risk factors associated with vertebral osteoporotic fractures in patients with rheumatoid arthritis. Clin Rheumatol. 2020;39:357-364. doi:10.1007/s10067-019-04787-9

8. Choi ST, Kwon SR, Jung JY, et al. Prevalence and fracture risk of osteoporosis in patients with rheumatoid arthritis: a multicenter comparative study of the FRAX and WHO criteria. J Clin Med. 2018;7:507. doi:10.3390/jcm7120507

9. Arain SR, Riaz A, Nazir L, Umer TP, Rasool T. Low bone mineral density among patients with newly diagnosed rheumatoid arthritis. $J$ Ayub Med Coll Abbottabad. 2016;28:175-178.

10. Coury F, Peyruchaud O, Machuca-Gayet I. Osteoimmunology of bone loss in inflammatory rheumatic diseases. Front Immunol. 2019;10:679. doi:10.3389/fimmu.2019.00679

11. Wilson JC, Sarsour K, Gale S, Pethö-Schramm A, Jick SS, Meier CR. Incidence and risk of glucocorticoid-associated adverse effects in patients with rheumatoid arthritis. Arthritis Care Res (Hoboken). 2019;1:498-511. doi:10.1002/acr.23611

12. Peng J, Gong Y, Zhang Y, Xiao Z, Zeng Q, Chen S. Bone mineral density in patients with rheumatoid arthritis and 4-year follow-up results. Clin Rheumatol. 2016;22:71-74. doi:10.1097/RHU.0000000000000359

13. Gabdulina G, Kasher M, Beissebayeva A, et al. An epidemiological analysis of osteoporotic characteristics in patients affected with rheumatoid arthritis in Kazakhstan. Arch Osteoporos. 2018;13:99. doi:10.1007/s11657-018-0514-9

14. Lee JH, Sung YK, Choi CB, et al. The frequency of and risk factors for osteoporosis in Korean patients with rheumatoid arthritis. $B M C$ Musculoskelet Disord. 2016;24:98. doi:10.1186/s12891-016-0952-8

15. Greco EA, Pietschmann P, Migliaccio S. Osteoporosis and sarcopenia increase frailty syndrome in the elderly. Front Endocrinol (Lausanne). 2019;10:255. doi:10.3389/fendo.2019.00255 
16. Cruz-Jentoft AJ, Baeyens JP, Bauer JM, et al. Sarcopenia: European consensus on definition and diagnosis: report of the European Working Group on sarcopenia in older people. Age Ageing. 2010;39:412-423. doi:10.1093/ageing/afq034

17. Barone M, Viggiani MT, Anelli MG, et al. Sarcopenia in patients with rheumatic diseases: prevalence and associated risk factors. $J$ Clin Med. 2018;7(12):504. doi:10.3390/jcm7120504

18. Dhillon RJ, Hasni S. Pathogenesis and management of sarcopenia. Clin Geriatr Med. 2017;33:17-26. doi:10.1016/j.cger.2016.08.002

19. Tada M, Yamada Y, Mandai K, Hidaka N. Matrix metalloprotease 3 is associated with sarcopenia in rheumatoid arthritis-results from the CHIKARA study. Int J Rheum Dis. 2018;21:1962-1969. doi:10.1111/1756-185X.13335

20. Torii M, Hashimoto M, Hanai A, et al. Prevalence and factors associated with sarcopenia in patients with rheumatoid arthritis. Mod Rheumatol. 2019;29:589-595. doi:10.1080/14397595.2018.1510565

21. Giles JT, Ling SM, Ferrucci L, et al. Abnormal body composition phenotypes in older rheumatoid arthritis patients: associated with disease characteristics an pharmacotherapies. Arthritis Rheum. 2018;59:907-915.

22. Doğan SC, Hizmetli S, Hayta E, Kaptanoğlu E, Erselcan T, Güler E. Sarcopenia in women with rheumatoid arthritis. Eur J Rheumatol. 2015;2:57-61. doi:10.5152/eurjrheum.2015.0038

23. Vlietstra L, Stebbings S, Meredith-Jones K, Abbott JH, Treharne GJ, Waters DL. Sarcopenia in osteoarthritis and rheumatoid arthritis: the association with self-reported fatigue, physical function and obesity. PLoS One. 2019;14:e0217462. doi:10.1371/journal.pone.0217462

24. Ngeuleu A, Allali F, Medrare L, Madhi A, Rkain H, Hajjaj-Hassouni N. Sarcopenia in rheumatoid arthritis: prevalence, influence of disease activity and associated factors. Rheumatol Int. 2017;37:1015-1020. doi:10.1007/s00296-017-3665-x

25. Tournadre A, Pereira B, Dutheil F, et al. Changes in body composition and metabolic profile during interleukin 6 inhibition in rheumatoid arthritis. J Cachexia Sarcopenia Muscle. 2017;8:639-646. doi:10.1002/jcsm.12189

26. Simon LS. DMARDs in the treatment of rheumatoid arthritis: current agents and future developments. Int J Clin Pract. 2000;54(4):243-249.

27. van Riel PL. The development of the disease activity score (DAS) and the disease activity score using 28 joint counts (DAS28). Clin Exp Rheumatol. 2014;32(5 Suppl 85):S-65-74.

28. van Riel PL, Renskers L. The Disease Activity Score (DAS) and the Disease Activity Score using 28 joint counts (DAS28) in the management of rheumatoid arthritis. Clin Exp Rheumatol. 2016;34(5 Suppl 101):S40-S44.

29. Joyce CR, Zutshi DW, Hrubes VF, Mason RM. Comparison of fixed interval and visual analogue scales for rating chronic pain. Eur $J$ Clin Pharmacol. 1975;8:415-420. doi:10.1007/BF00562315

30. Jensen MP, Chen C, Brugger AM. Interpretation of visual analog scale ratings and change scores: a reanalysis of two clinical trials of postoperative pain. J Pain. 2003;4:407-414. doi:10.1016/S1526-5900(03)00716-8

31. Soen S, Fukunaga M, Sugimoto T, et al. Diagnostic criteria for primary osteoporosis: year 2012 revision. J Bone Miner Metab. 2013;31:247-257. doi:10.1007/s00774-013-0447-8

32. Brown BH, Karatzas T, Nakielny R, Clarke RG. Determination of upper arm muscle and fat areas using electrical impedance measurements. Clin Phys Physiol Meas. 1988;9(1):47-55. doi:10.1088/0143-0815/9/1/004

33. Pietrobelli A, Morini P, Battistini N, Chiumello G, Nuñez C, Heymsfield SB. Appendicular skeletal muscle mass: prediction from multiple frequency segmental bioimpedance analysis. Eur J Clin Nutr. 1998;52(7):507-511. doi:10.1038/sj.ejen.1600592

34. Chen LK, Liu LK, Woo J, et al. Sarcopenia in Asia: consensus report of the Asian Working Group for Sarcopenia. J Am Med Dir Assoc. 2004;15:95-101. doi:10.1016/j.jamda.2013.11.025

35. Mobini M, Kashi Z, Ghobadifaret A. Prevalence and associated factors of osteoporosis in female patients with rheumatoid arthritis. Caspian $J$ Intern Med. 2012;3:447-450.

36. Kweon SM, Sohn DH, Park JH, et al. Male patients with rheumatoid arthritis have an increased risk of osteoporosis. Medicine (Baltimore). 2018;97:e11122. doi:10.1097/MD.0000000000011122

37. Yamada Y, Tada M, Mandai K, Hidaka N, Inui K, Nakamura H. Glucocorticoid use is an independent risk factor for developing sarcopenia in patients with rheumatoid arthritis: from the CHIKARA study. Clin Rheumatol. 2020;39(6):1757-1764. doi:10.1007/s10067-020-04929-4

38. Matsumoto H, Tanimura C, Tanishima S, Osaki M, Noma H, Hagino H. Sarcopenia is a risk factor for falling in independently living Japanese older adults: a 2-year prospective cohort study of the GAINA study. Geriatr Gerontol Int. 2017;17:2124-2130. doi:10.1111/ggi.13047

39. Chalhoub D, Cawthon PM, Ensrud KE, et al. Risk of nonspine fracture in older adults with sarcopenia low bone mass or both. J Am Geriatr Soc. 2015;63:1733-1740. doi:10.1111/jgs.13605

40. Clynes MA, Edwards MH, Buehring B, Dennison EM, Binkley N, Cooper C. Definitions of sarcopenia: associations with previous falls and fracture in a population sample. Calcif Tissue Int. 2015;97:445-452. doi:10.1007/s00223-015-0044-z

41. Yoon BH, Lee JK, Choi DS, Han SH. Prevalence and associated risk factor of sarcopenia in female patients with osteoporotic fracture. $J$ Bone Metal. 2018;25:59-62. doi:10.11005/jbm.2018.25.1.59

42. Lee DW, Choi EY, Wu Q. Sarcopenia as an independent risk factor for decreased BMD in COPD patients: Korean National Health and Nutrition Examination Surveys IV and V(2008-2011). PLoS One. 2016;11:e0164303. doi:10.1371/journal.pone.0164303

43. Di Monaco M, Vallero F, Di Monaco R, Tappero R. Prevalence of sarcopenia and its association with osteoporosis in 313 older women following a hip fracture. Arch Gerontol Geriatr. 2011;52:71-74. doi:10.1016/j.archger.2010.02.002

44. Liu X, Hou L, Xia X, et al. Prevalence of sarcopenia in multi ethnics adults and the association with cognitive impairment: findings from West-China health and aging trend study. BMC Geriatr. 2020;20:63. doi:10.1186/s12877-020-1468-5

45. Roubenoff R, Rall RC. Rheumatoid cachexia: metabolic abnormalities, mechanisms and interventions. Rheumatology. 2004;43:1219-1223. doi:10.1093/rheumatology/keh321

46. Roubenoff R, Roubenoff RA, Cannon JG, et al. Rheumatoid cachexia: cytokine-driven hypermetabolism accompanying reduced body cell mass in chronic inflammation. J Clin Invest. 1994;93:2379-2386. doi:10.1172/JCI117244

47. Gupta S, Dhillon RJS, Hasni S. Sarcopenia: a rheumatic disease? Rheum Dis Clin North Am. 2018;44(3):393-404. doi:10.1016/j.rdc.2018.03.001 


\section{Publish your work in this journal}

The International Journal of General Medicine is an international, peer-reviewed open-access journal that focuses on general and internal medicine, pathogenesis, epidemiology, diagnosis, monitoring and treatment protocols. The journal is characterized by the rapid reporting of reviews, original research and clinical studies across all disease areas. The manuscript management system is completely online and includes a very quick and fair peer-review system, which is all easy to use. Visit http://www.dovepress.com/testimonials.php to read real quotes from published authors.

Submit your manuscript here: https://www.dovepress.com/international-journal-of-general-medicine-journal 